\title{
Monitoring of Culicoides at 20 locations in northwest Germany
}

\author{
Ellen Kiel • Gabriele Liebisch • René Focke • \\ Arndt Liebisch • Doreen Werner
}

Published online: 12 May 2009

(C) Springer-Verlag 2009

\section{Erratum to: Parasitol Res \\ DOI 10.1007/s00436-009-1409-x}

The original version of this article unfortunately contained a mistake. Doreen Werner was not listed among the authors. Please note the correct list of authors is Ellen Kiel, Gabriele Liebisch, René Focke, Arndt Liebisch and Doreen Werner.

The online version of the original article can be found at http://dx.doi. org/10.1007/s00436-009-1409-x.

E. Kiel $(\bowtie) \cdot$ R. Focke

Carl von Ossietzky Universität Oldenburg,

Campus Haarentor,

26111 Oldenburg, 0049-441-4719, Germany

e-mail: ellen.kiel@uni-oldenburg.de

R. Focke

e-mail: Rene.focke@uni-oldenburg.de

G. Liebisch $(\bowtie) \cdot$ A. Liebisch

ZeckLab, Labor für klinische Diagnostik und Prüfung,

Up'n Kampe 3,

30938 Burgwedel, Germany

e-mail: liebisch@zecklab.de

D. Werner

Leibniz-Zentrum für Agrarlandschaftsforschung (ZALF) e.V.,

Senckenberg Deutsches Entomologisches Institut,

Eberswalder Straße 90,

15374 Müncheberg, Germany

e-mail: dwerner@zalf.de 